\title{
La flore bactérienne des eaux thermales et minérales
}

\author{
M. Capdepuy, J. Canellas \\ Université de Bordeaux 2 \\ Laboratoire de Microbiologie Pharmaceutique
}

Comme la plupart des écosystèmes dans lesquels ou à côté desquels évolue l'homme, les eaux minérales et thermales ne sont pas stériles. Elle sont l'habitat d'une flore bactérienne dont l'identité et la physiologie dépendent des caractères physico-chimiques de l'environnement (température, $\mathrm{pH}$, potentiel rédox des eaux, présence de composés organiques ou inorganiques pour n'en citer que quelquesuns). Chaque eau minérale représente donc un écosystème particulier et héberge une flore bactérienne propre qui s'adapte à ses conditions spécifiques, mais qui à son tour agit sur elles.

Un élément semble relativement constant pour les eaux minérales en l'absence de contamination : il s'agit de leur très faible teneur en matière organique. Elles n'hébergeront donc que très peu de bactéries dites chimioorganotrophes, qui ont besoin de cet aliment pour se développer. Le nombre des bactéries possédant ce type métabolique diminue avec le taux de matière organique, lui même lié, le plus souvent, à la profondeur des nappes.

\section{LES BACTÉRIES CHIMIOLITHOTRO- PHES}

Mais une autre catégorie de bactéries, les chimiolithotrophes, est capable de tirer son énergie de l'utilisation (sou- vent par oxydation) de composés minéraux : on les trouvera donc dans les forages profonds. Ces bactéries sont moins étudiées dans les eaux minérales que les chimioorganotrophes car leur culture est plus longue et plus délicate et elles ne sont pas pathogènes pour l'homme. Mais il ne faut pas sous-estimer leur impact car elles interviennent souvent de façon décisive dans les principaux cycles géochimiques (Carbone, Azote, Soufre et Fer) en oxydant ou réduisant successivement ces éléments.

Dans les eaux sulfurées, par exemple, on voit apparaittre sur les bassins d'émergence ou sur les canalisations, des formations blanches abondantes que l'on appelle bioglées ou glairines, et auxquelles on associe parfois le nom de la station (Barégine, Luchonine...). Il s'agit d'ensembles complexes de bactéries, virus, végétaux, voire animaux, vivant en symbiose. Dans le cas de Luchon, nous voyons qu'il s'agit essentiellement de bactéries qui oxydent les sulfures: il s'agit de Beggiatoa, qui sont des bactéries filamenteuses qui accumulent le soufre élémentaire sous forme de granules réfringents à l'intérieur des cellules. On retrouve également dans ces catégories de bactéries les Thiotrix et Thiobacillus qui sont des petits bacilles à gram négatif, de types physiologiques très divers ; certains peuvent se développer à des $\mathrm{pH}$ très acides, d'autres à des températures élevées [1] [2].

\section{Bacterial flora in thermal and mineral waters}

Mineral and thermal waters are not sterile, but contain an indigenous microbial flora of a kind related to the physical and chemical characteristics of these biotopes.

This microflora can be divided into two groups on a metabolic basis : first, lithotrophic bacteria which draw their energy from inorganic substances, and secondly organotrophic bacteria which use organic matter. The former play an important role in the cycle of elements such as sulphur and iron, and in the formulation of certain thermal compounds such as glairins, but have been little studied owing to the slowness of their growth on synthetic media. The second group, which can be demonstrated by simple and rapid methods of culture, consist essentially of gram-negative bacilli (Pseudomonas, Xanthomonas, Acinetobacter, etc.) and show high numerical stability with time. They have no health significance but any sudden rise in their number can indicate contamination of the resource.

Better knowledge of these two types of bacterial flora could make it possible to select biological identity markers from the species characteristic of the resource and lead to a better understanding of their role in the operation of this natural ecosystem. 
Ces bioglées peuvent dans certains cas servir d'adjuvants pour la confection de boues thérapeutiques. Mais les bactéries du soufre peuvent être à l'origine d'effets indésirables, en particulier de la corrosion. A l'heure actuelle, l'importance de leur participation à ce phénomène est encore mal évaluée mais on sait par exemple que les bacilles sulfato-réducteurs participent à des phénomènes électrochimiques sur des surfaces métalliques, ou bien que certains sulfoxydants produisent directement des substances corrosives type $\mathrm{H}_{2} \mathrm{SO}_{4}[3]$.

Dans le cas des eaux ferrugineuses, les bactéries du cycle du Fer (Gallionella, Leptothrix) seront prédominantes : ces bactéries peuvent oxyder les ions ferreux, et aussi parfois les ions manganeux. Elles aussi, lorsqu'elles se développent de façon exagérée, peuvent entraîner des phénomènes d'altération de l'eau ou bien des réseaux.

\section{LES BACTÉRIES CHIMIOORGANO- TROPHES}

La flore organotrophe est composée surtout de bacilles à Gram négatif (Pseudomonas, Xanthomonas, Acinetobacter...) et son étude permet une évaluation de la qualité sanitaire des eaux au plan microbiologique. Les analyses de surveillance [4] préconisent la numération de la flore aérobie mésophile revivifiable c'est-à-dire la numération des bactéries capables de se développer après incubation à $22{ }^{\circ} \mathrm{C}(72 \mathrm{~h})$ et à $37^{\circ} \mathrm{C}(24 \mathrm{~h})$, sur un milieu gélosé enrichi en matières organiques (extrait de levure, peptones...). Cette méthode présente l'avantage d'être facile à mettre en cuvre.

Le chiffre retrouvé n'a pas de signification particulière en valeur absolue car des études [5] ont montré que ces bactéries ne pouvaient pas s'implanter dans le tube digestif mais ce nombre semble constant dans le temps, et c'est la raison pour laquelle une brusque augmentation doit constituer un signal d'alarme. Dans ce cas une contamination de l'écosystème a dû intervenir et il faut en mesurer la portée sanitaire. II n'existe donc pas de recommandation chiffrée, sauf pour les eaux minérales destinées à être embouteillées, pour lesquelles il existe une limite quantitative de $100 \mathrm{UFC} / \mathrm{ml}$ à $22^{\circ} \mathrm{C}-72$ h et $20 \mathrm{UFC} / \mathrm{ml}$ à $37^{\circ} \mathrm{C}$ $-24 \mathrm{~h}$, après 12 heures d'embouteillage (UFC: Unités Formant Colonies) [6].

Cela suppose des chiffres très bas avant embouteillage (soit $20 \mathrm{UFC} / \mathrm{ml}$ à $22^{\circ} \mathrm{C}$ et $5 \mathrm{UFC} / \mathrm{ml}$ à $37^{\circ} \mathrm{C}$ ) qui limiteront la prolifération bactérienne peu souhaitable car les produits du métabolisme bactérien peuvent être toxiques même lorsqu'il ne s'agit pas de bactéries pathogènes. Il a en effet été prouvé que l'embouteillage des eaux plates permettait une augmentation très significative, mais transitoire, de la flore totale. Ce phénomène est beaucoup plus limité, voire inexistant, dans les eaux carbogazeuses à cause de l'action inhibitrice du gaz carbonique sur la croissance bactérienne.

La recherche de certaines bactéries témoins (Coliformes totaux (CT), coliformes thermotolérants (CF), streptocoques fécaux (SF) et anaérobies sporulés sulfito-réducteurs (ASR)) permet d'évaluer les contaminations d'origine fécale susceptibles de polluer la ressource.

Quelle que soit l'utilisation ultérieure de l'eau minérale, l'eau doit en être dépourvue. Il n'y a que dans les piscines thermales, en l'absence de traitement de désinfection, que ces bactéries sont retrouvées. Mais une hygiène rigoureuse antérieure aux bains, permet de limiter ce phénomène.

Bien d'autres bactéries chimioorganotrophes peuvent être retrouvées dans les eaux minérales et thermales mais deux ont retenu l'attention des hygiénistes en raison de leur caractère pathogène opportuniste. Elles représentent une menace pour la santé de personnes fragilisées, dont le potentiel immunitaire est diminué. Il s'agit de Pseudomonas aeruginosa et de Legionella pneumophila.

Pseudomonas aeruginosa est un bacille à gram négatif retrouvé très fréquemment dans les environnements hydriques et parfois même dans l'intestin humain. Cette bactérie présente une remarquable aptitude à se multiplier dans l'eau, même apparemment dépourvue de nutriments, ainsi que dans toutes sortes de substrats (tartre, joint de canalisation, voire même certains antiseptiques type ammonium quaternaire). Elle s'adapte à une large gamme de température $\left(10\right.$ à $\left.40^{\circ} \mathrm{C}\right)$ et participe activement à l'élaboration de biofilms grâce à la production d'exopolysaccharides. Ces biofilms constituent une protection contre l'action des désinfectants et permettent des recroissances bactériennes indésirables.

L'absence de Pseudomonas aeruginosa est donc fortement recommandée pour les eaux minérales destinées à l'ingestion et à la plupart des soins.

Mais l'eau minérale est l'habitat de beaucoup d'autres espèces de Pseudomonas et de bactéries à Gram négatif apparentées comme le montre une étude de HUNTER [7]. On peut difficilement les exclure mais déjà certaines espèces sont responsables d'infections en milieu hospitalier. Il convient donc d'être vigilant sans être alarmiste.

Legionella pneumophila est une bactérie qui fait aussi partie de la flore des eaux douces (elle n'a pas été jusqu'alors isolée des eaux de mer) et qui affectionne les températures de $40-45^{\circ} \mathrm{C}$. Sa transmission à l'homme se fait plutôt par voie aérienne et occasionne des pneumopathies graves à caractère épidémique ou endémique comme dans certains hôpitaux.

Il existe des formes plus bénignes comme la fièvre de Pontiac, d'allure grippale. Les procédés d'aérosolisation à partir d'eaux thermales (surtout sulfurées) représentent donc un danger potentiel, compte tenu de l'écologie particulière de cette bactérie.

Des études menées en 1988 par l'équipe du Professeur FLEURETTE et de Madame BORNSTEIN [8] ont montré qu'il $\mathrm{y}$ avait effectivement des Légionelles dans les eaux thermales à des concentrations allant de $10^{3}$ à $10^{5}$ UFC/l et qu'elles appartiennent à au moins 15 espèces avec une nette prédominance de Legionella pneumophila. Le danger potentiel représenté par ces bactéries nécessite d'être vigilant quant au nombre de ces bactéries ( $100 \mathrm{UFC} / \mathrm{l}$ semble une limite souhaitable) et aux mesures d'hygiène pouvant freiner leur prolifération.

En particulier, il faut éviter toute stagnation dans les réseaux, ainsi que la formation des biofilms.

Toutes ces informations pourraient laisser croire qu'il est périlleux d'entreprendre des cures thermales : ce n'est bien évidemment pas le cas ; les curistes constatent plutôt des effets bénéfiques. Il faut bien rappeler que ces risques concernent surtout les sujets fragilisés : le bulletin de l'Ordre des médecins a d'ailleurs rappelé que la prescription d'une cure thermale devait tenir compte de l'état général du sujet. 
Quant aux eaux embouteillées, elles n'ont jamais été à l'origine d'épidémie sauf au Portugal en 1974 où 2467 cas de choléra causant 28 décès ont été recensés [9]. Vibrio cholerae avait été isolé de la source qui était mal protégée au point de vue géologique et avait été contaminée par des eaux usées.

L'eau est le plus souvent d'excellente qualité à la ressource, mais une attention toute particulière doit être portée lors des opérations ultérieures (notamment d'embouteillage) et à la protection des sites. Des dégradations de la qualité microbiologique peuvent intervenir à ce niveau.

Ces aspects sanitaires mis à part, la connaissance plus approfondie de la flore spécifique de chaque source est indispensable car elle fait partie du contrôle d'identité du produit. Ce dernier est un élément indispensable d'évaluation de la qualité et doit, dans les années qui viennent, être développé pour renforcer ce qu'il convient de nommer, comme pour les industries pharmaceutiques, les « bonnes pratiques de fabrication».

\section{Bibliographie}

[1] HARrISSON A.P. (1984), - The acidophilic thiobacilli and other acidophilic bacteria that share their habitat. Ann. Rev. Microbiol., 38, pp. 265-295.

[2] Wood A.P. et Kelly D.P. (1985). - Physiological characteristics of a new thermophilic obligately chemolithotrophic
Thiobacillus sp. : Thiobacillus tepidarius. International Journal of Systematic Bacteriology, 35, n 4, pp. 434-437.

[3] Little B., Wagner P. et Mansfeld F. (1992). - An overview of microbiology influenced corrosion. Electrochimica acta, $37, \mathrm{n}^{\circ} 12$, pp. 2185-2194.

[4] Annexe $\mathrm{n}^{\circ} 1$ de la circulaire DGS/SD1 D/92 $\mathrm{n}^{\circ} 513 \mathrm{du}$ 20 juillet 1992.

[5] Ducluzeau R., Dufresne S. et Bochand J.M. (1976). Inoculation of the digestive tract of axenic mice with the autochtonous bacteria of mineral water. European Journal of Applied Microbiology, 2, pp. 127-134.

[6] European Community (1980). - Council directive $\mathrm{n}^{\circ} 80$ (777) E.E.C. of 15 july 1980 . On the approximation of the laws of the member states relating to the exploitation and marketing of natural mineral waters. Official Journal of the European Communities L.29, 1 octobre 1980.

[7] HUNTER P.R. (1993). - The microbiology of bottled natural mineral waters. Journal of Applied Bacteriology, 74, pp. 345352.

[8] Bornstein N. Fleurette J. et coll. (1988). - Risque de Legionelloses chez les curistes fréquentant un établissement thermal. La lettre de l'infectiologue, $4, \mathrm{n}^{\circ} 12$.

[9] Blake P.A. et coll. (1977). - Cholera in Portugal. Transmission by bottled mineral waters. American Journal of Epidemiology, 105, pp. 344-348. 